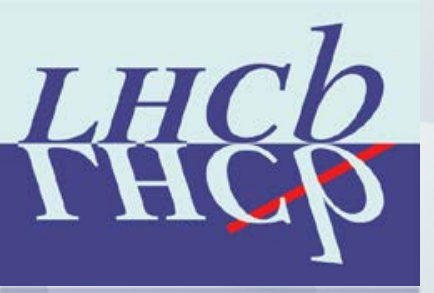

\title{
Calibration of the $\mathrm{LHCb}$ calorimetric system
}

\author{
Dmitrii Pereima \\ on behalf of the $\mathrm{LHCb}$ collaboration \\ Institute for Theoretical and Experimental Physics \\ NRC «Kurchatov Institute», Moscow, Russia \\ Instrumentation for Colliding Beam Physics \\ 27 February - 3 March 2017, Novosibirsk, Russia
}




\section{$\begin{array}{ll}\text { LHCb } & \text { LHCb calorimeter system }\end{array}$}

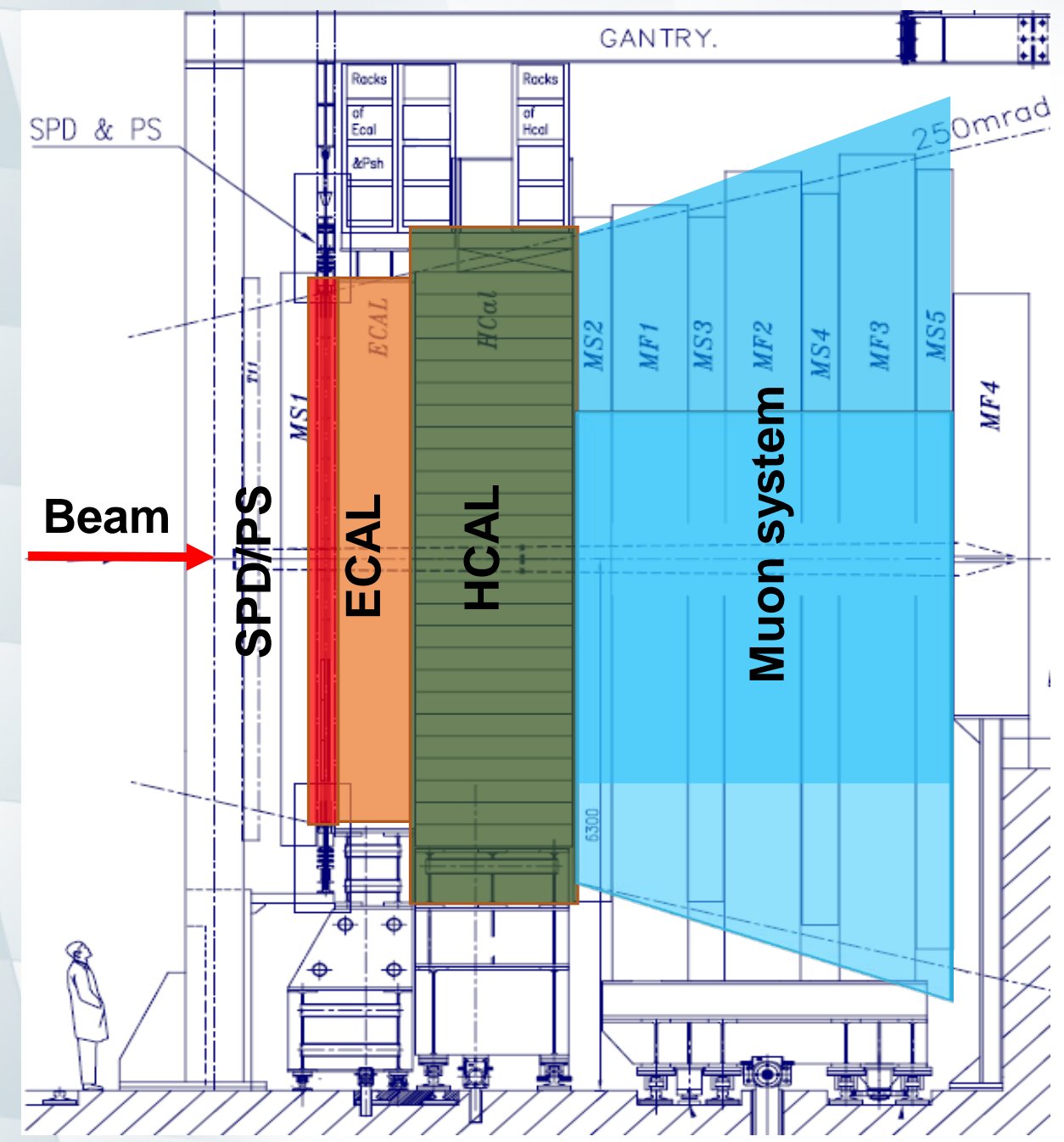

Fig. 1: General view of the $\mathrm{LHCb}$ calorimeter and muon system
Calorimeter system consists of:

- scintillator-pad detector (SPD);

- preshower detector (PS);

- electromagnetic calorimeter (ECAL);

- hadronic calorimeter (HCAL)

\section{Main goals:}

- $\quad$ to provide Level-0 (L0) trigger;

- particle identification and energy reconstruction

- More details on design of the $\mathrm{LHCb}$ calorimeter system: talk of Yuri Guz 


\section{$\begin{array}{ll}\text { KHCp } & \text { SPD and PS }\end{array}$}

\section{SPD/PS description:}

- Structure:

- Scintillator Pad - lead plane - Scintillator Pad;

- Light collection and readout:

- WLS - fibers

- 64-channel multi - anode PMT HAMAMATSU R7600

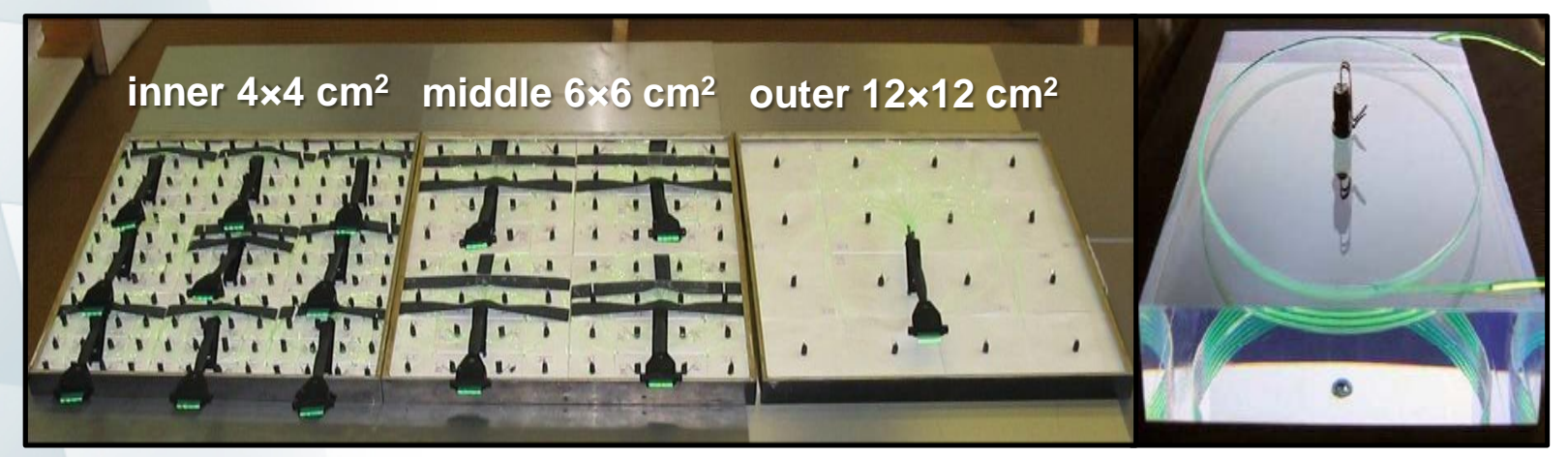

Fig. 2: Photo of the SPD/PS modules

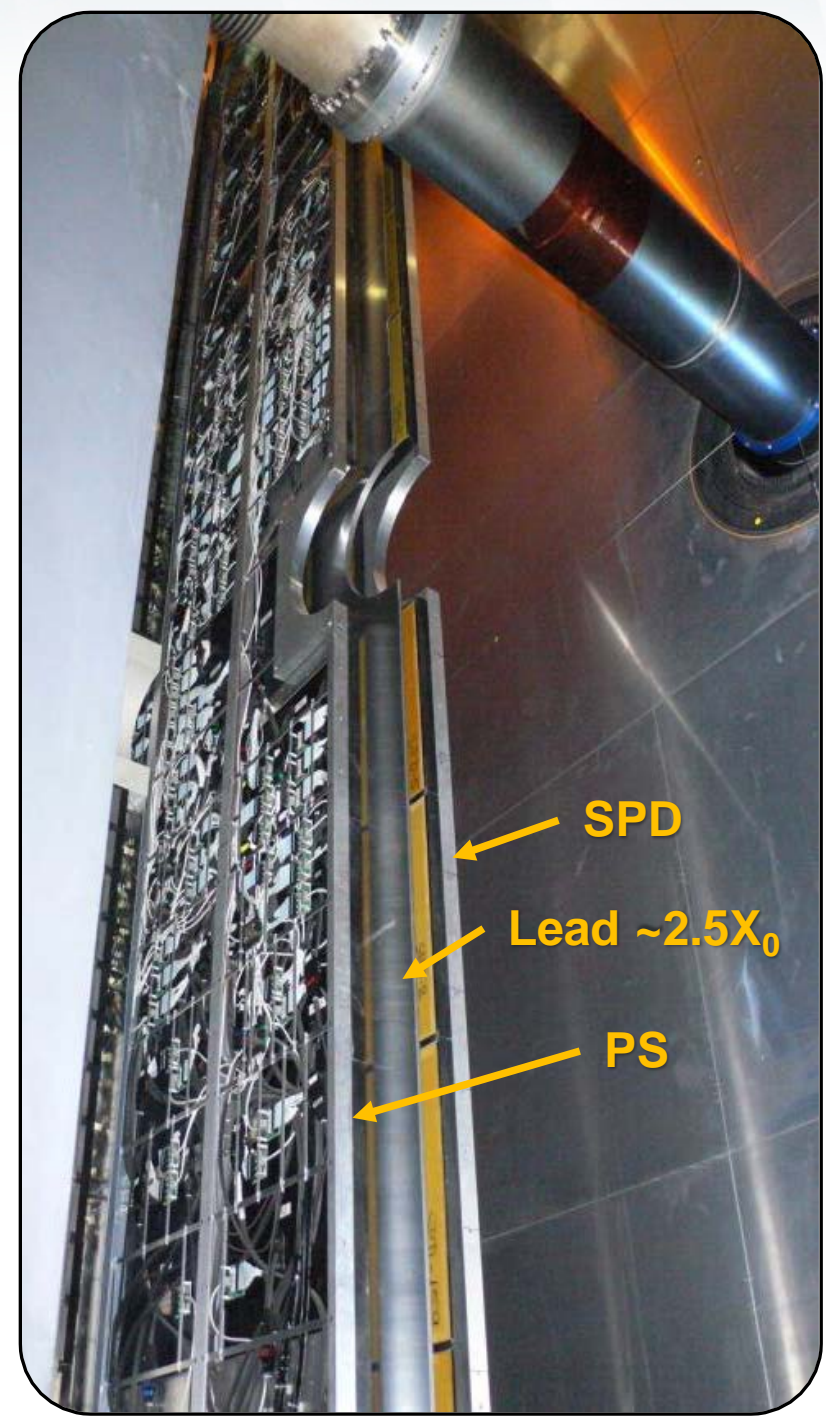

Fig. 3: Photo of the SPD and PS 


\section{KHCD}

ECAL description:

- performed in «shashlik» technology;

- subdivided into three zones: outer (1), middle (2) and inner (3);

- number of detection cells depending on zone

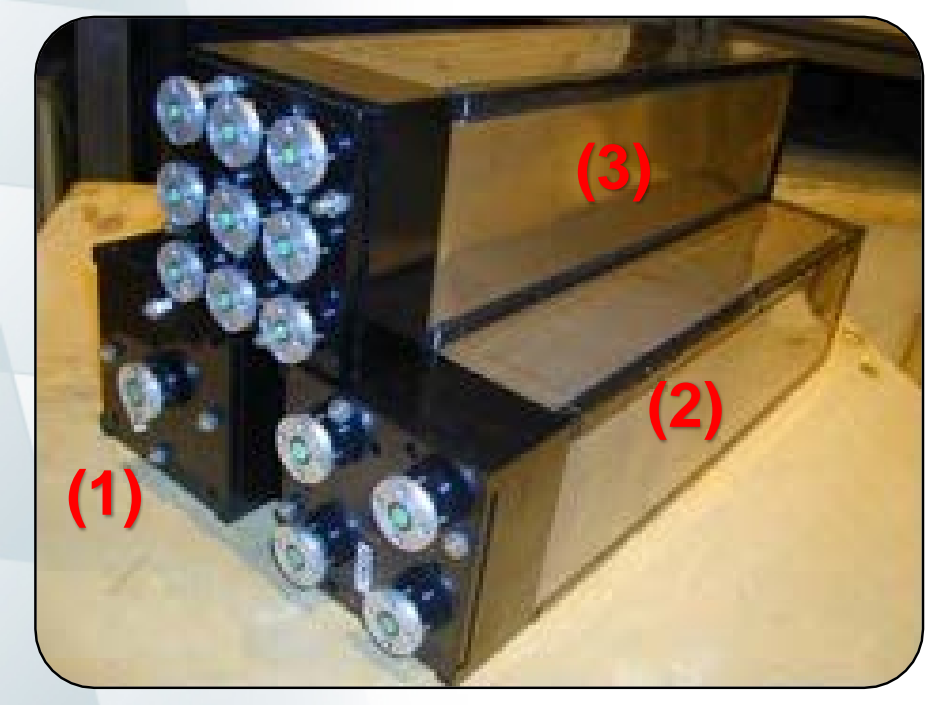

Fig. 4: Photo of the ECAL modules for three sections
Energy resolution:

$$
\frac{\sigma_{E}}{E}=\frac{(8.5 \div 9.5) \%}{\sqrt{E}} \oplus 0.8 \% \text {, }
$$

where $E$ - energy in $\mathrm{GeV}$

$$
\text { [2008 JINST } 3 \text { S08005] }
$$

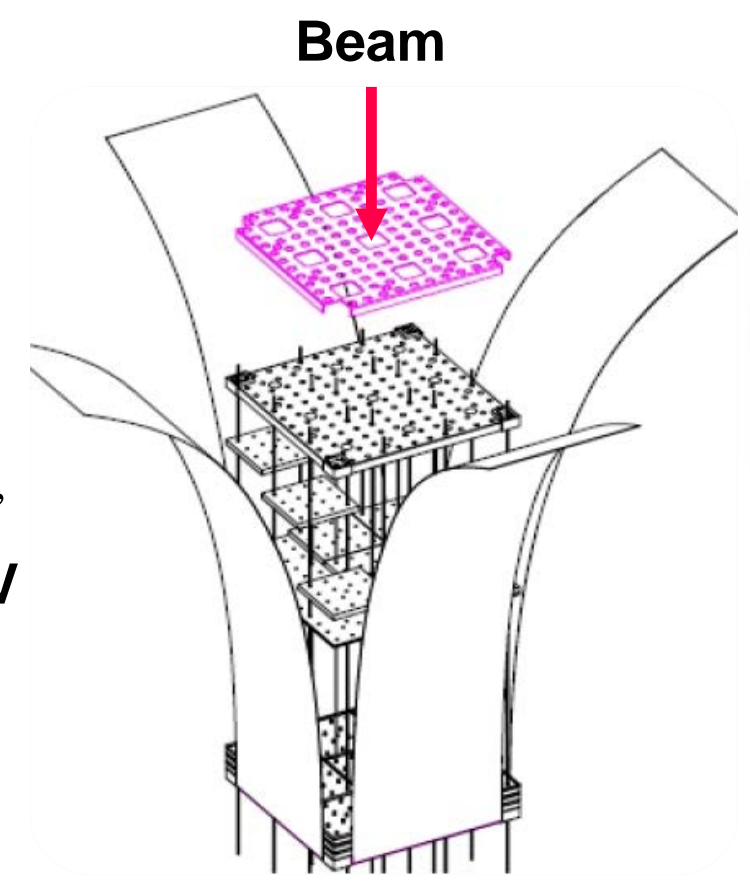

Fig. 5: Inner structure of the ECAL module 


\section{LHCb
HACP}

HCAL description:

- performed in the ATLAS TileCal technology;

- two symmetrical halves, system is subdivided into inner and outer zones;

- detection cells are subdivided into six sections in longitudinal direction

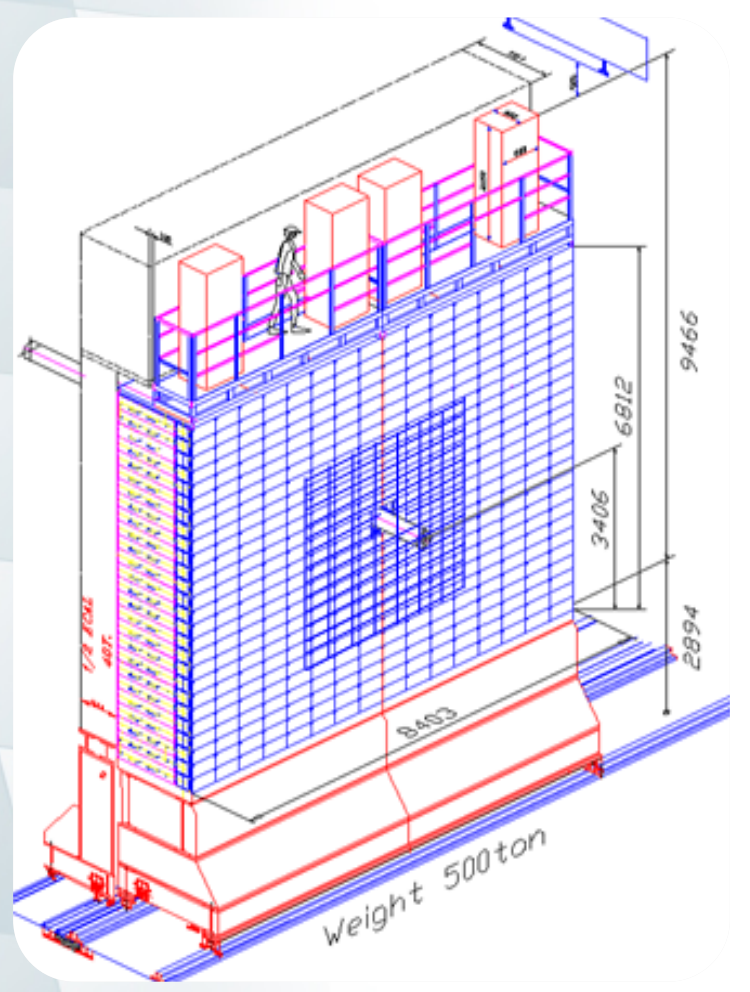

Fig. 6: Schematic view of the HCAL

\section{Energy resolution:}

$$
\frac{\sigma_{E}}{E}=\frac{(69 \pm 5) \%}{\sqrt{E}} \oplus(9 \pm 2) \%,
$$

where $E$ - energy in $\mathrm{GeV}$

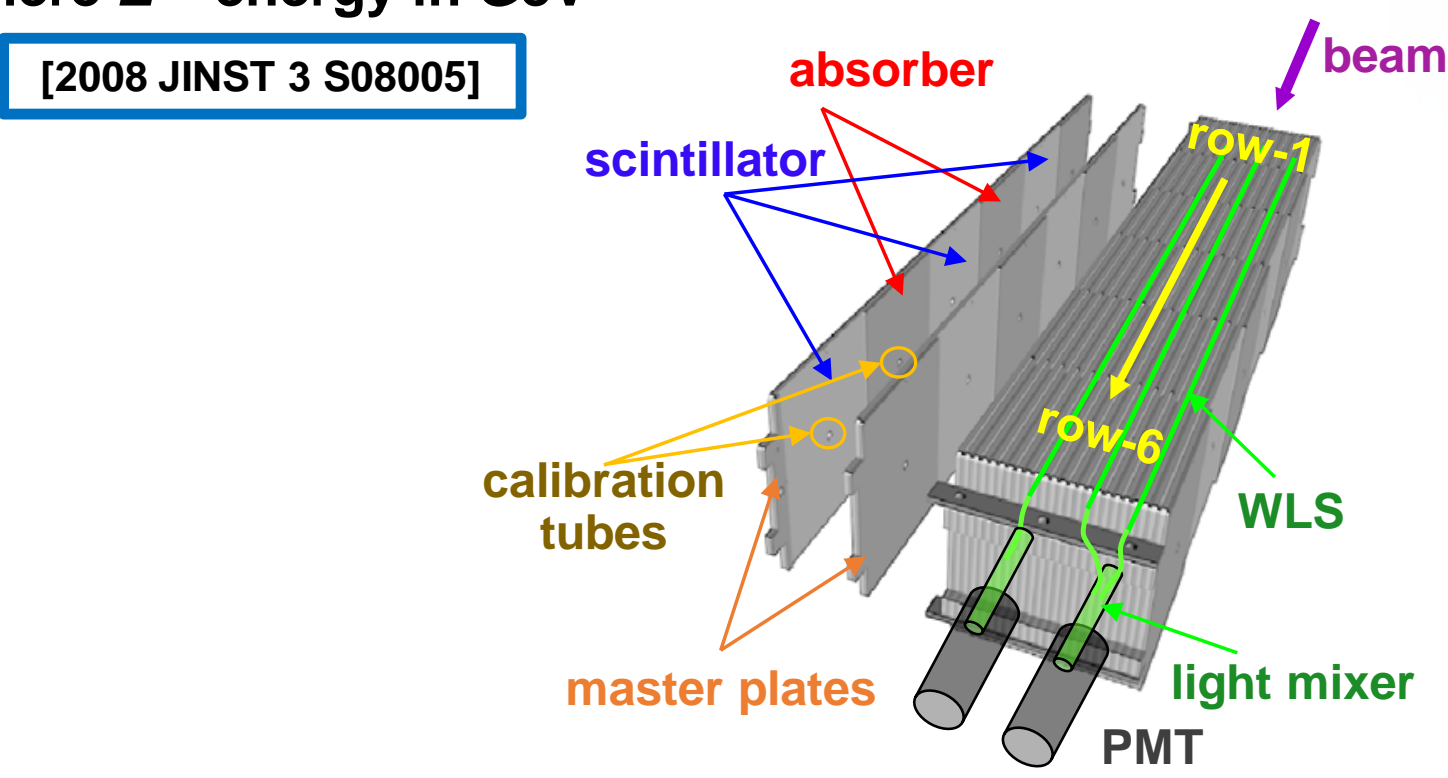

Fig. 7: Inner structure of the HCAL cell 


\section{LHCD Calorimeter system functionalities}

PS/SPD particle identification for LO photon and electron trigger:

- $\mathrm{e}, \pi^{0}, \gamma$ separation by PS;

- $\gamma /$ MIP separation by SPD;

- charged multiplicity by SPD

\section{ECAL:}

- high $E_{T}$ electrons, photons and $\pi^{0}$ for L0 trigger;

- reconstruction of $\pi^{0}$ and photons (+ PS);

- particle ID (+ PS)

HCAL:

- high $\mathrm{E}_{\mathrm{T}}$ hadrons;

- contributes to Muon ID;

- provides $\sim 70 \%$ of LO trigger output (500 kHz out of $\sim 1 \mathrm{MHz}$ )

Table 1: Coincidence of the calorimeter system

\begin{tabular}{|c|c|c|c|c|}
\hline SPD & PS & ECAL & HCAL & \\
\hline 1 & 1 & 1 & 0 & e \\
\hline 0 & 1 & 1 & 0 & $\gamma$ \\
\hline 1 & 0 & 0 & 1 & h \\
\hline
\end{tabular}

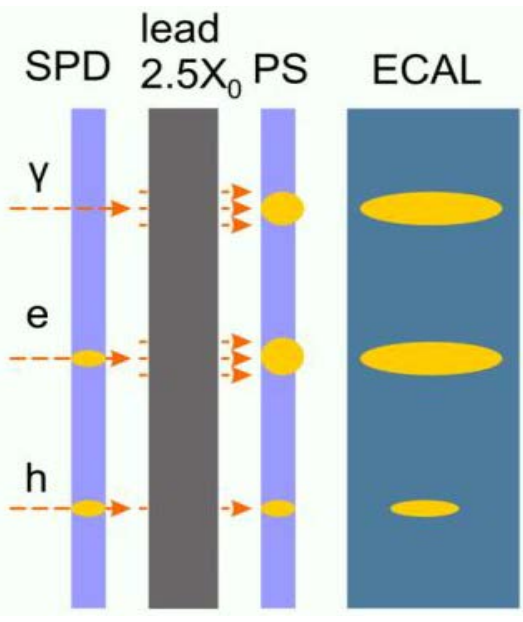




\section{LHCb Calorimeter system parameter summary}

Table 2: Selected parameters of calorimeter system

\begin{tabular}{|c|c|c|c|}
\hline Sub-detector & SPD/PS & ECAL & HCAL \\
\hline Number of channels & $2 \times 6016$ & 6016 & 1488 \\
\hline Lateral size & $6.2 \times 7.6 \mathrm{~m}^{2}$ & $6.3 \times 7.8 \mathrm{~m}^{2}$ & $6.8 \times 8.4 \mathrm{~m}^{2}$ \\
\hline $\begin{array}{l}\text { cell size in } \mathrm{mm}: \\
\text { - inner } \quad \text { middle } \cdot \text { outer }\end{array}$ & $\begin{array}{l}\text { SPD(PS): 39.66(39.84); } \\
\text { 59.5(59.76); 119(119.5) }\end{array}$ & $40.4 ; 60.6 ; 121.2$ & 131.3; (no middle section);262.6 \\
\hline Longitudinal depth & $180 \mathrm{~mm}-2.5 \mathrm{X}_{0}-0.1 \lambda_{\mathrm{I}}$ & $835 \mathrm{~mm}-25 \mathrm{X}_{0}-1.1 \lambda_{\mathrm{I}}$ & $1655 \mathrm{~mm}-5.6 \lambda_{\mathrm{I}}$ \\
\hline Light yield & 20 ph.el./MIP & 3000 ph.el./GeV & 105 ph.el./GeV \\
\hline Basic requirement & Average light yield $\sim 20$ ph.el./MIP & $\frac{(8.5 \div 9.5) \%}{\sqrt{E}} \oplus 0.8 \%$ & $\frac{\sigma_{E}}{E}=\frac{(69 \pm 5) \%}{\sqrt{E}} \oplus(9 \pm 2) \%$ \\
\hline Dynamic range & $\begin{array}{c}0-100 \text { MIPs }-1 \text { bit (SPD) } \\
10 \text { bits (PS) }\end{array}$ & $E^{\max }=10+7 \cdot \sin \Theta$ & $E^{\max }=30 / \sin \Theta$ \\
\hline
\end{tabular}

ECAL and HCAL dynamic range adjusted in each cell according to cells: $\sin \Theta=\sqrt{x^{2}+y^{2}} / \sqrt{x^{2}+y^{2}+z^{2}}$ 


\section{Method:}

- Initial calibration

- energy flow method

- $\pi^{0}$ meson reconstruction
Accuracy:

- 10\%

- $\sim 5 \%$

- $\sim 2-2.5 \%$
Periodicity:

- before LHC startup

- with the first data ìn 2010

- every month
Stability of PMT gains is monitored each 15 minutes by an LED system. Corrections are applied automatically.
[Int. J. Mod. Phys. A 30 (2015) 1530022] a)

$$
\begin{aligned}
& -1 \\
& 3 \\
& 3
\end{aligned}
$$

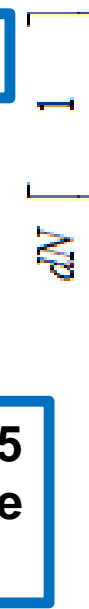

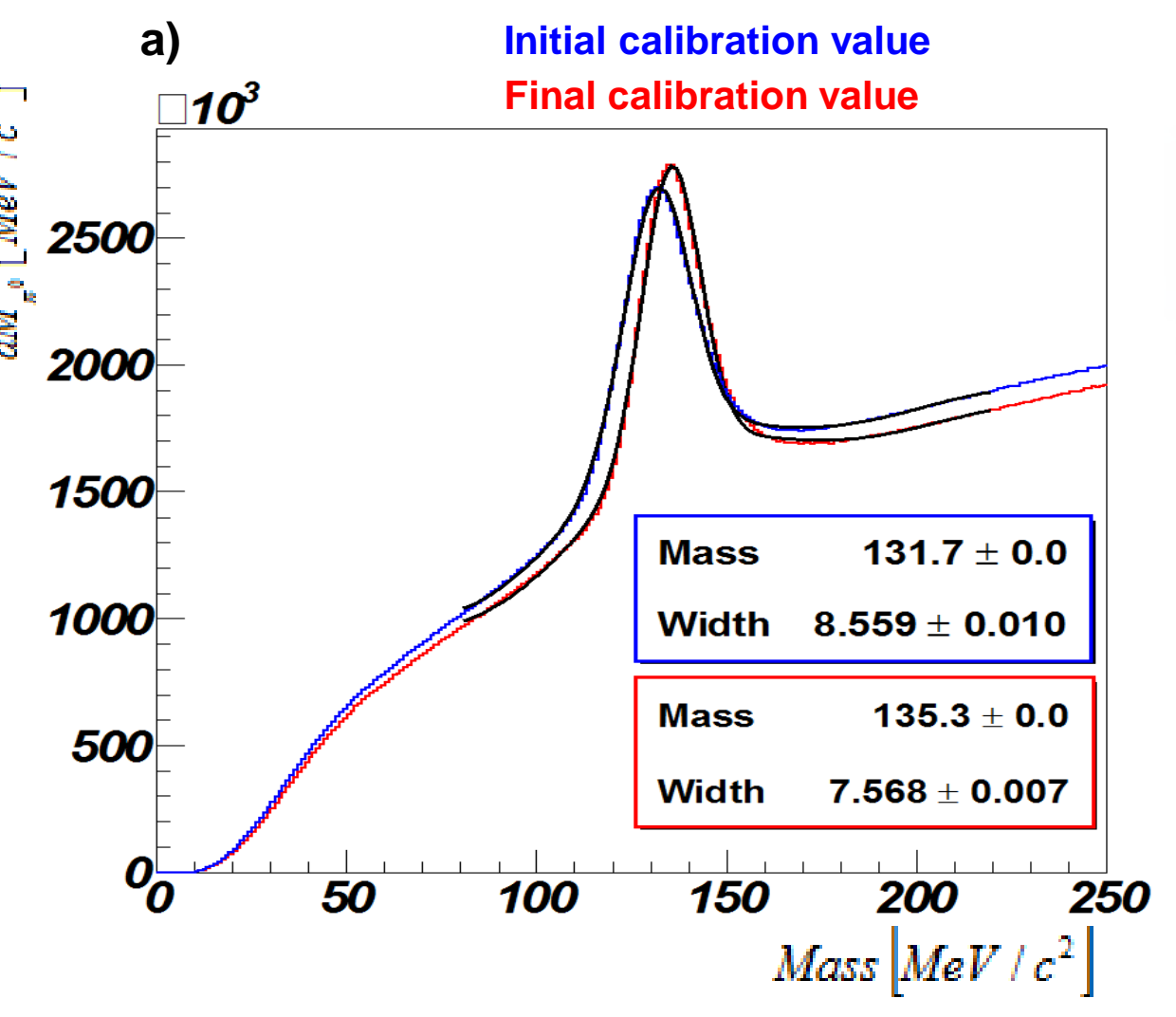

Fig. 8: Distribution of $\Pi^{0}$-meson invariant mass 


\section{LHCb ECAL $\pi^{0}$ invariant mass calibration}

The fine absolute calibration method uses reconstructed $\pi^{0}$ meson invariant mass:

- allows to achieve the accuracy of calibration of $2 \%$

Step 1: photon reconstruction by the standard experiment algorithms

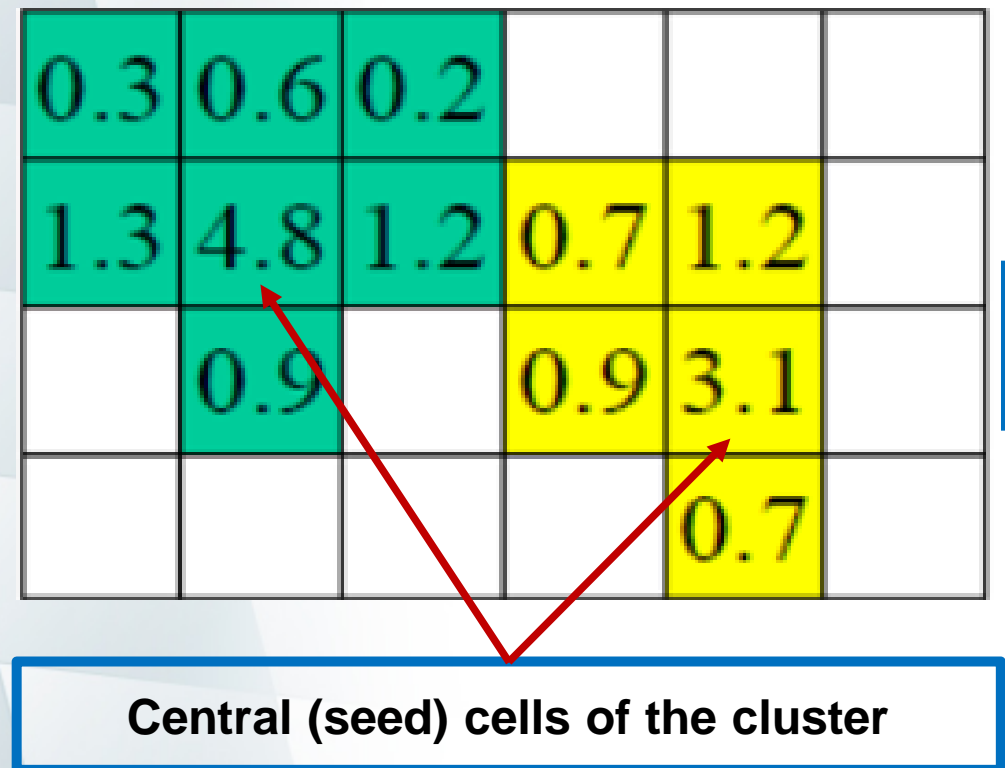

Fig. 9: Energy deposition in the cluster cells
Reconstructed photon energy:

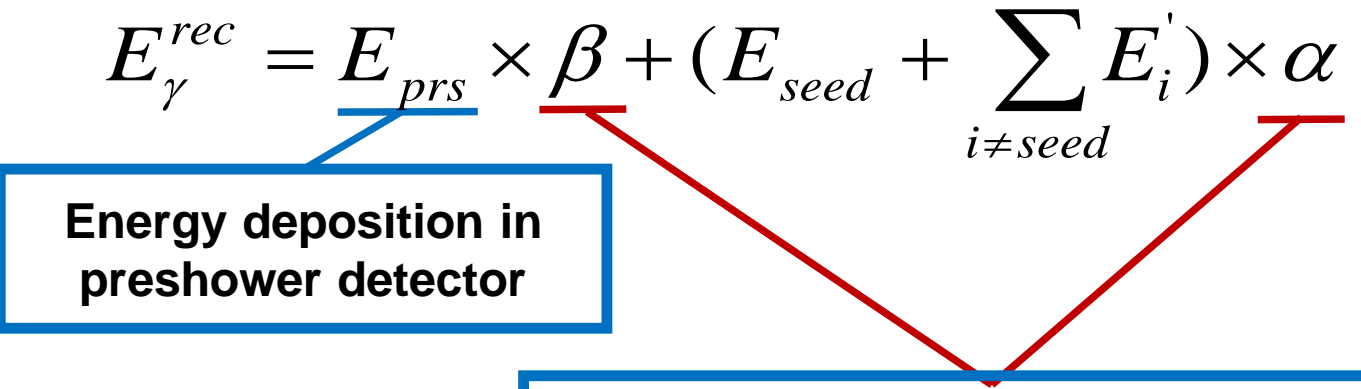

Out of the shower outside the cluster Moliere radius $3.5 \mathrm{~cm}$ Cluster

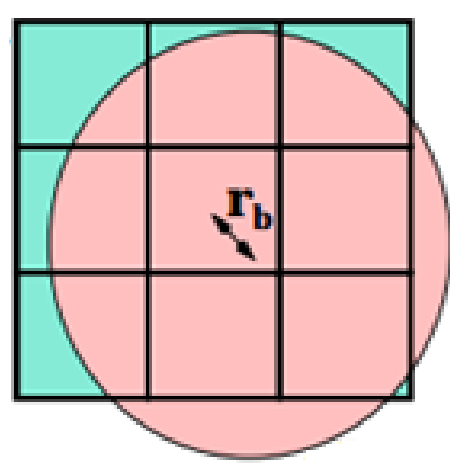

Photon shower reconstructed photon energy is substantially defined by the energy of the seed cell 


\section{LHCb ECAL $\pi^{0}$ invariant mass calibration}

Step 2: selection of photon pairs, which form $\pi^{0}$-candidate

Algorithm in C++ language

$$
\begin{aligned}
& M_{\pi^{0}}^{2}=2 E_{1}^{\gamma} E_{2}^{\gamma}\left(1-\underline{\cos \theta_{\gamma \gamma}}\right) \\
& \text { Photons } \\
& \text { energies } \\
& \text { Opening angle } \\
& \text { between photons }
\end{aligned}
$$

\section{For all ECAL cells}

Step 3: select photon pairs, for which the cell is the central cell of the cluster

Step 4: determination of the shift of $\pi^{0}-$ meson mass from the nominal value in the given cell

Program modules ìn Python language

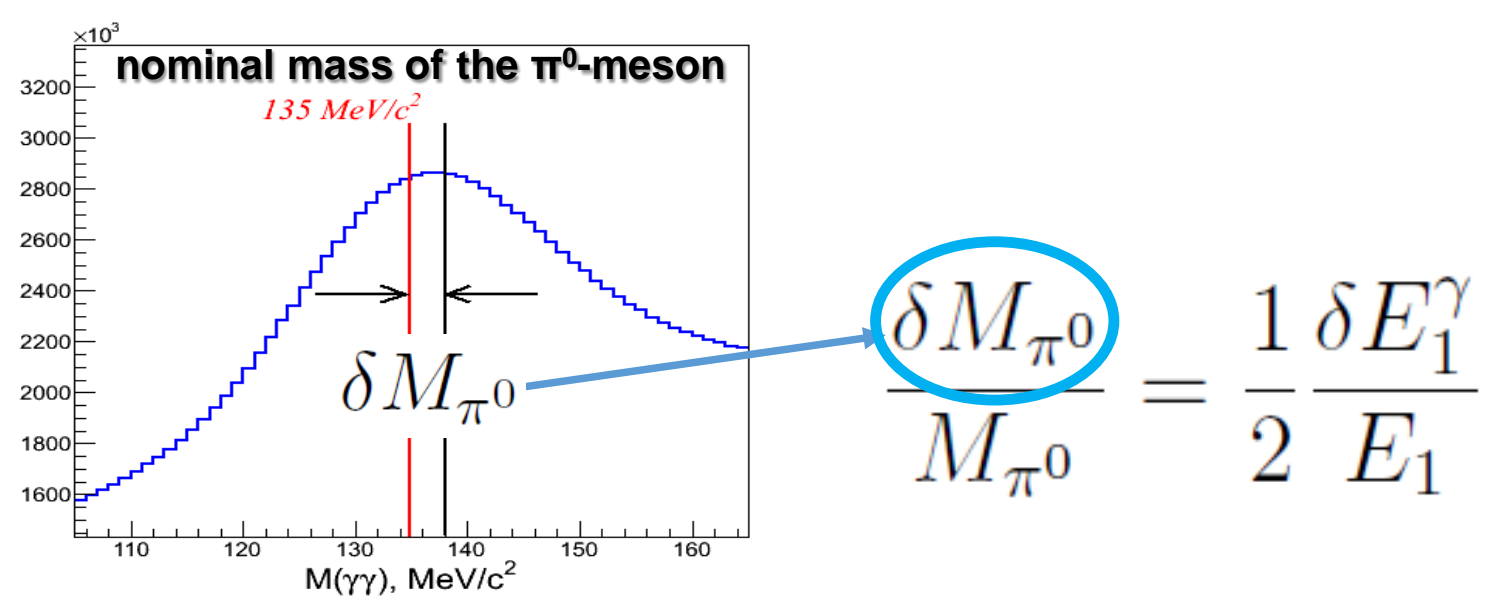

Fig. 10: Distribution of $\pi^{0}-$ meson invariant mass 


\section{KHCP ECAL $\pi^{0}$ invariant mass calibration}

Step 5: estimation of the calibration coefficient:

Step 6: «primary» iterations

$$
\lambda=1.0-\frac{\delta M_{\pi^{0}}}{M_{\pi^{0}}}
$$

Program modules in

Python language

Program modules in Python language

To correct for the fact that not all photon energy is deposited in the central cell of the cluster

\section{$\Pi^{0}$-meson ìnvariant mass}

a)

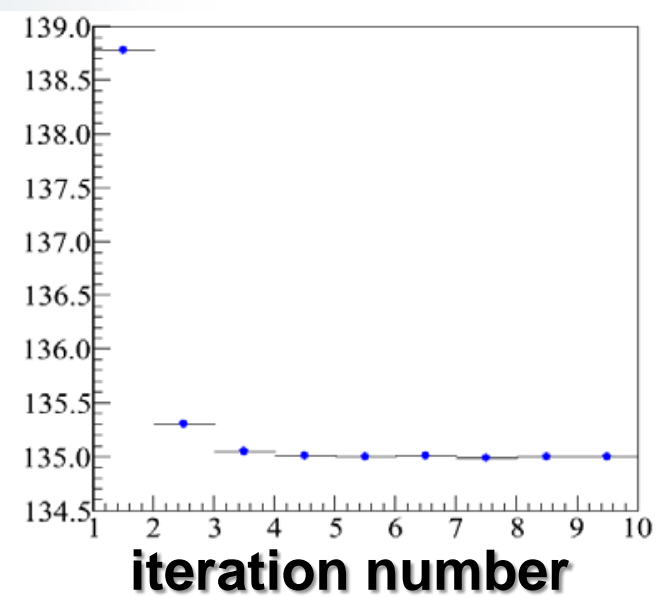

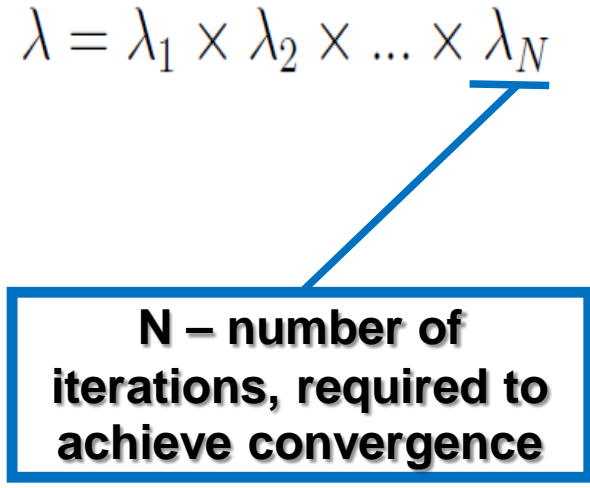

mass resolution of $\pi^{0}$-meson

b)

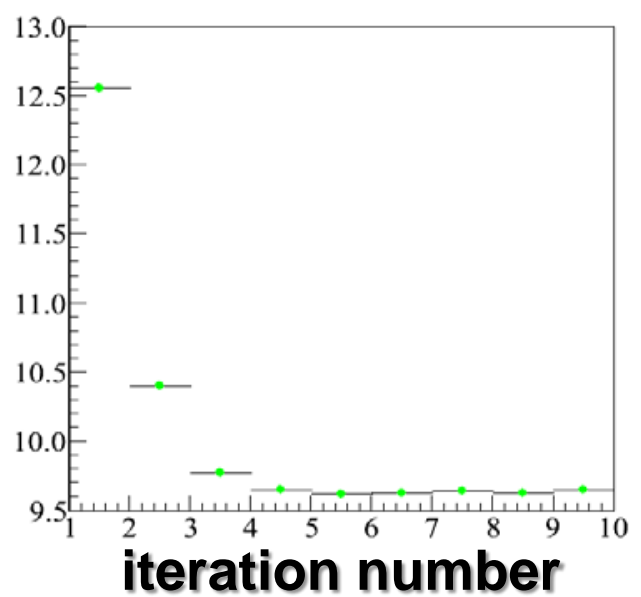

Fig. 11: Dependences of $\pi^{0}$ invariant mass (a) and mass resolution (b) on the number of iterations Step 7: «secondary» iterations

Algorithm in $\mathbf{C + +}$ language

To account for the fact, that after applying calibration coefficients the cluster positions may change. After the third "secondary" iteration the values of majority of the coefficients vary by no more than $1 \%$ 
HCAL calibration is performed with ${ }^{137} \mathrm{Cs}$ source:

- similar to the ATLAS TileCal system

- two $\sim 10 \mathrm{mCi}{ }^{137} \mathrm{Cs}$ sources used (1 per each detector half);

\section{Features:}

- allows to measure the response of every scintillating tile;

- absolute normalization $\sim \mathbf{1 0 \%}$ (the accuracy of the source activity measurements)

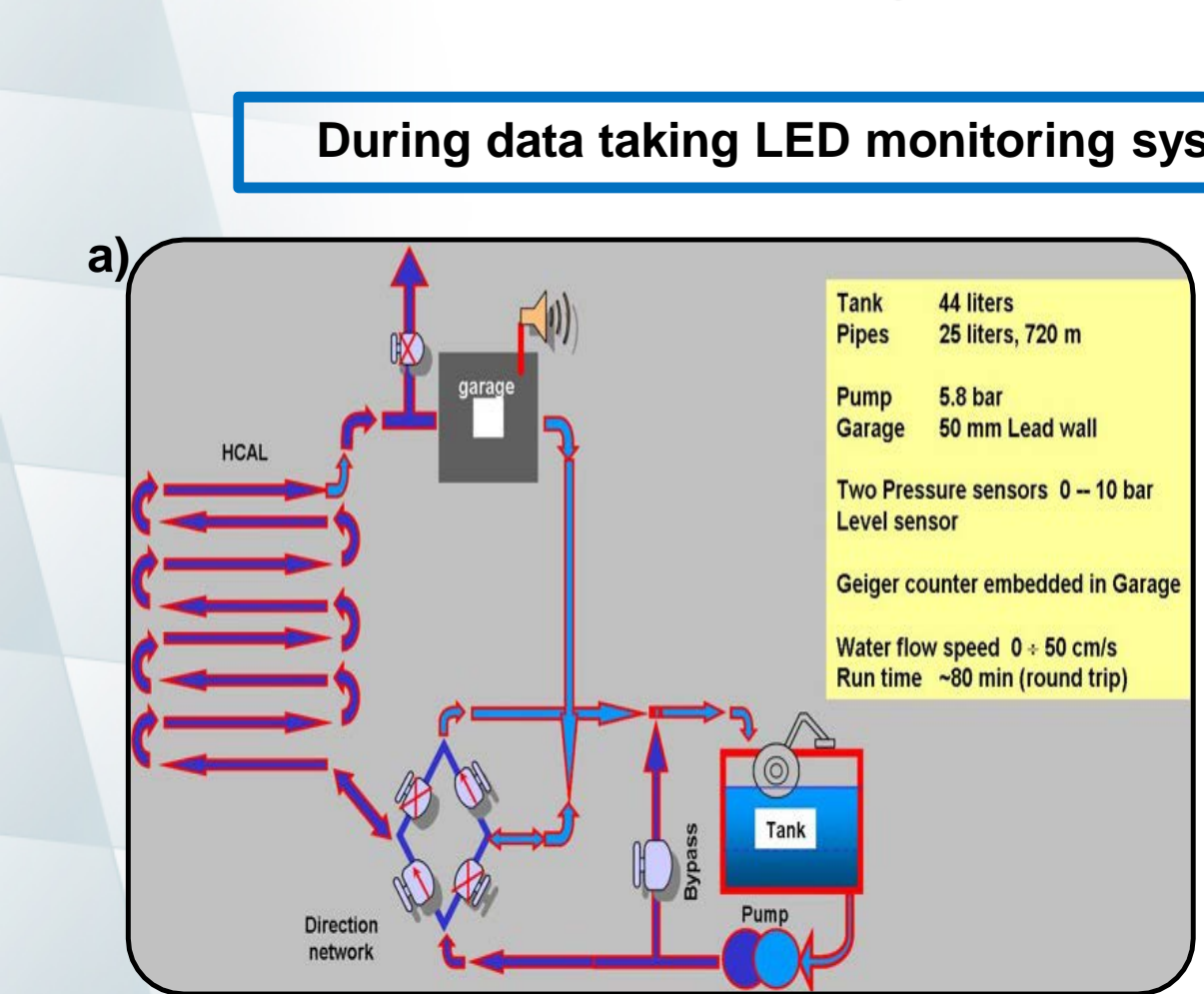

b)
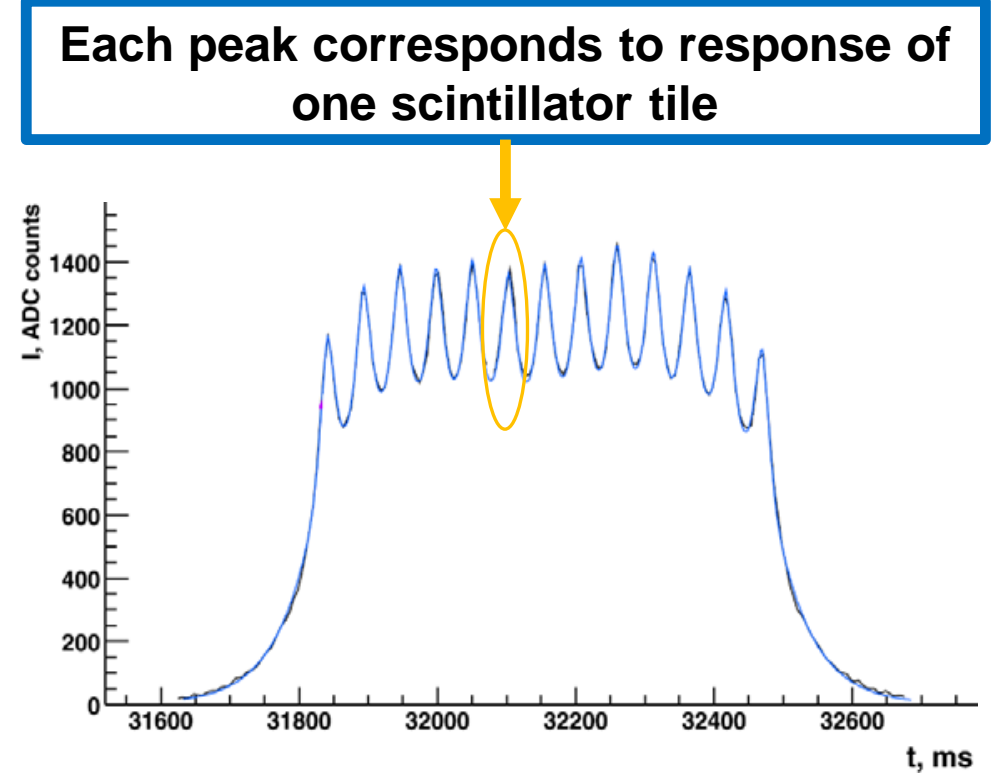

Fig. 12: Sketch of hydraulic system (a), PMT anode current as a function of run time (b) 


\section{LHCb $\quad{ }^{137} \mathrm{Cs}$ source movement system}

Each module has a $\sim 27 \mathrm{~m}$ embedded six-fold pipe. The pipe passes through the centers of each tile row. All modules are connected together.

- between calibrations the source is housed inside lead container (so-called garage), the hydraulic pipe system is integrated into it;

- the source is driven by a pump, a system of valves determines the direction of water flow;

- average source speed $\sim 30 \mathrm{~cm} / \mathrm{sec}$;

- calibration data taking is performed for both capsule movement directions

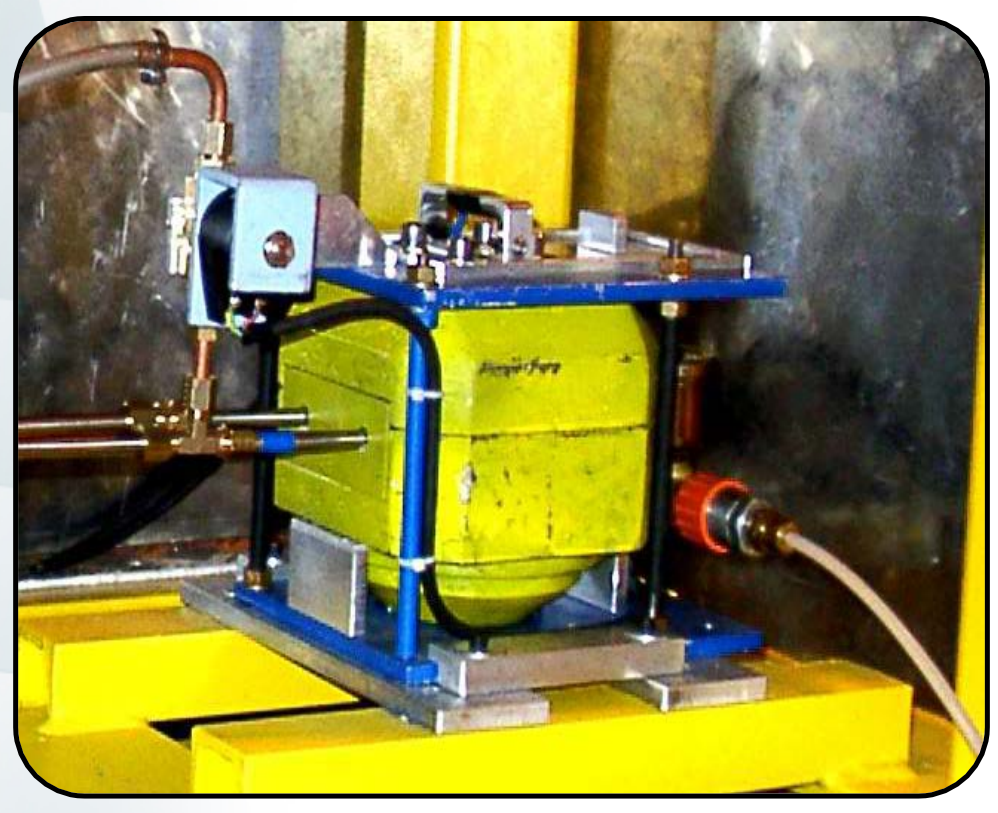

Fig. 13: Photo of the garage

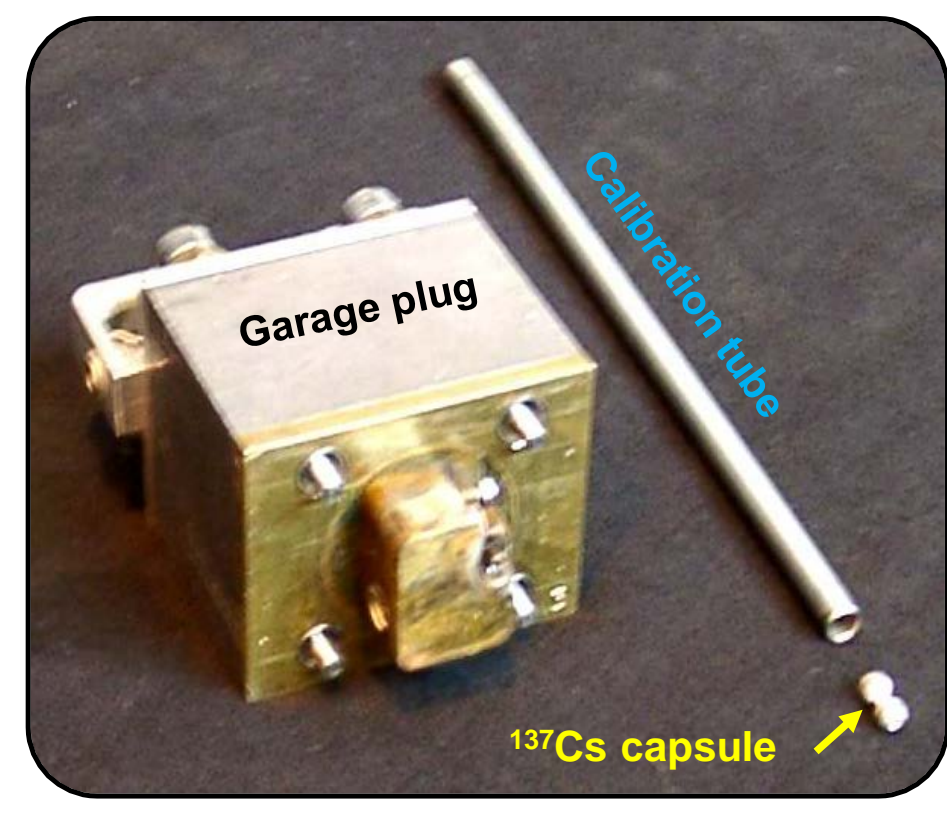

Fig. 14: Hydraulic system elements 


\section{LHCD HCAL ${ }^{137} \mathrm{Cs}$ calibration beam test results}

The precision of the ${ }^{137} \mathrm{Cs}$ calibration was studied at the $50 \mathrm{GeV} \pi-$ beam in 2003 at SPS X7. Independent calibrations coincide within $2-3 \%$. The ratio of sensitivities to ${ }^{137} \mathrm{Cs}$ Y - radiation to hadrons and scintillator light yield was measured:

$\kappa_{C s}=41.07(20.88)\left[\frac{\mathrm{nA} / \mathrm{mCi}}{p C / G e V}\right] \begin{aligned} & - \text { ratio of the sensitivities to }{ }^{137} \mathrm{Cs} \text { and hadronic shower } \\ & \text { for outer (inner) cells }\end{aligned}$ $P_{h}=105 \pm 5\left[\frac{\mathrm{ph} . \mathrm{el} .}{\mathrm{GeV}}\right]$ - scintillator light yield

\section{[LHCb note 2003-143]}

a)

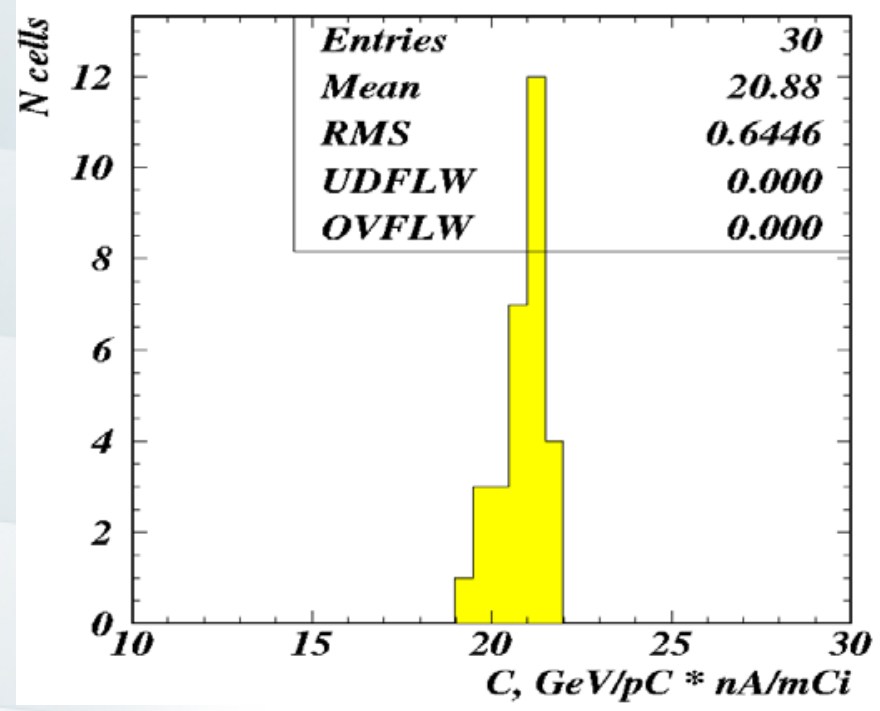

b)

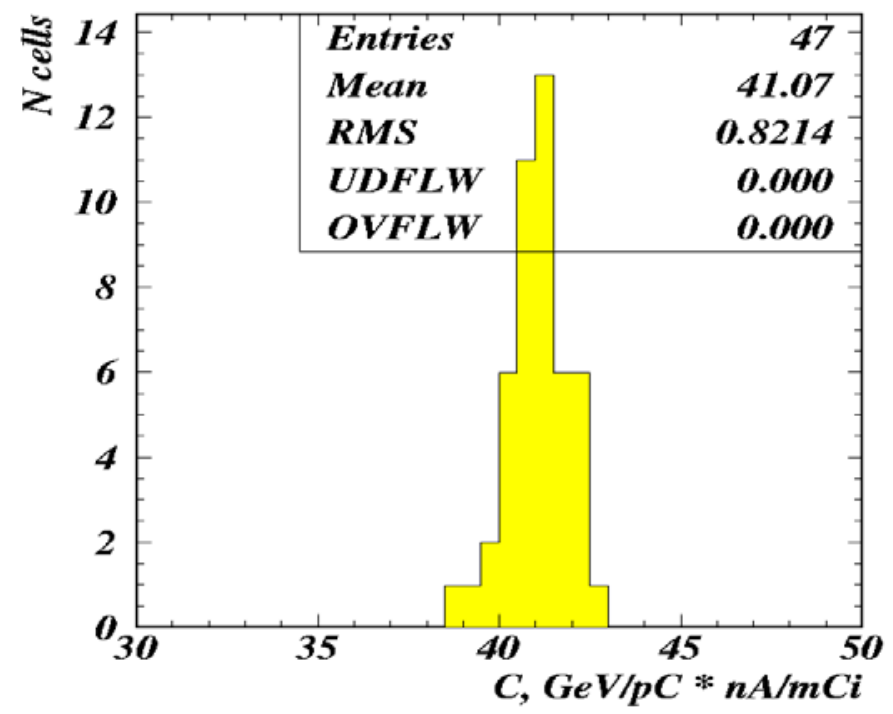

Fig. 15: The distribution of the ratio $S_{C S} / S_{\pi}$ for inner (a) and outer (b) cells 


\section{HCAL ${ }^{137}$ Cs calibration data}

Calibration data from 14.09.2016: measured PMT anode currents, illustrates the nominal calibration

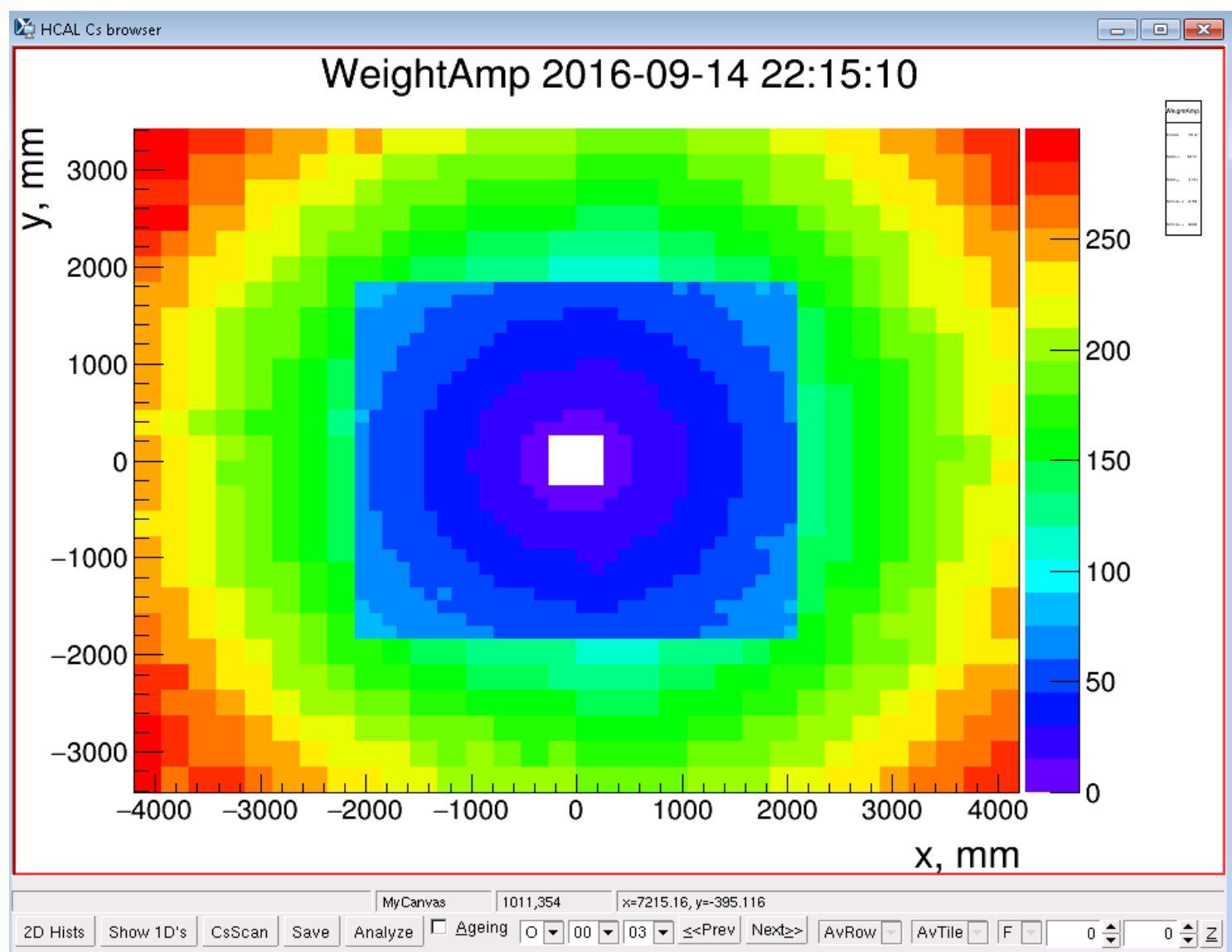

Fig. 16: Calibration results (map of average PMT anode currents) 


\section{KHCb HCAL ${ }^{137}$ Cs calibration multipass runs}

The effective regulation curves can be obtained from several sequential ${ }^{137} \mathrm{Cs}$ runs at different PMT HV settings. Multipass calibration is carried out once a year.

A PMT regulation curve could be parameterized in the form:

$$
G^{e f f}=G_{0}^{e f f} \cdot H V^{\alpha}
$$

$G^{\text {eff }}$ - determined under assumption that the light yield of the cell is $\sim 100 \mathrm{ph} . \mathrm{el} / \mathrm{GeV}$

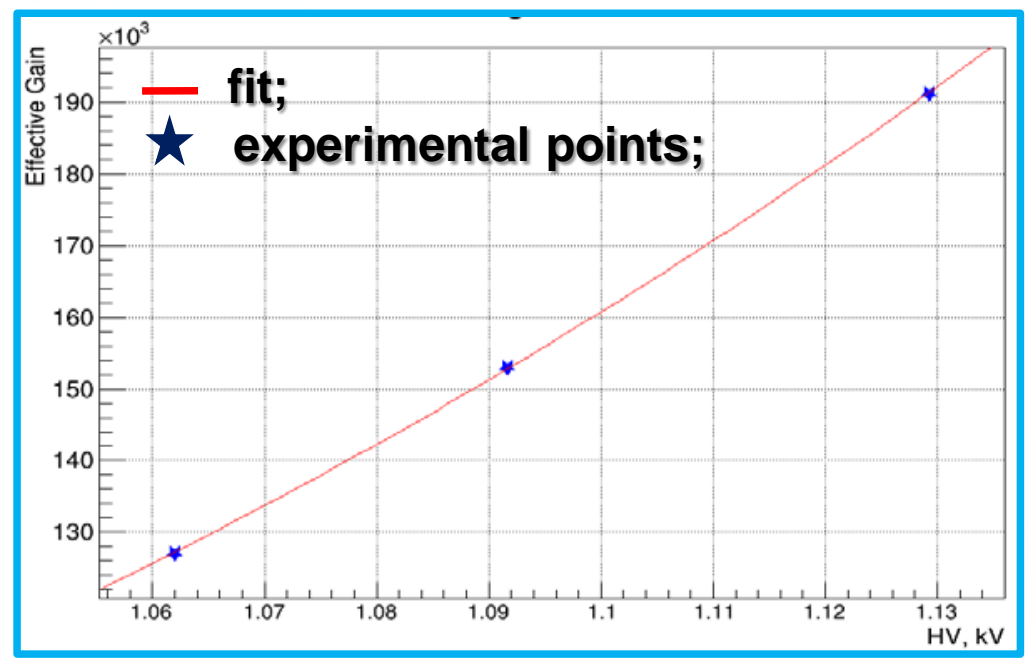

Fig. 17: Typical view of the PMT regulation curve

a)

The main goal is to obtain PMT regulation curve parameters, $G_{0}$ and $\alpha$, at the $H V$ range around the expected working point $( \pm 20 \%)$.

The a parameter is then used to calculate HV corrections

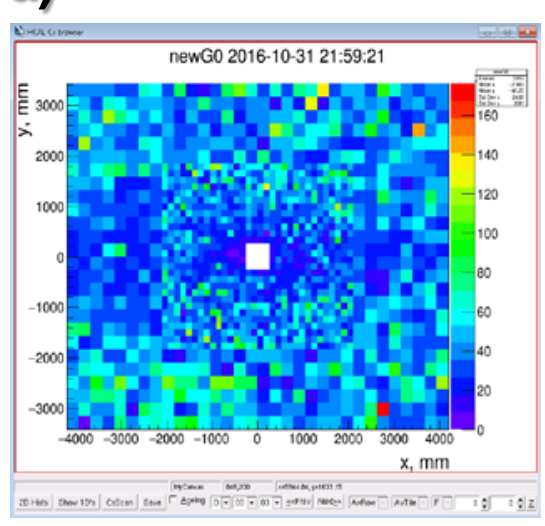

b)

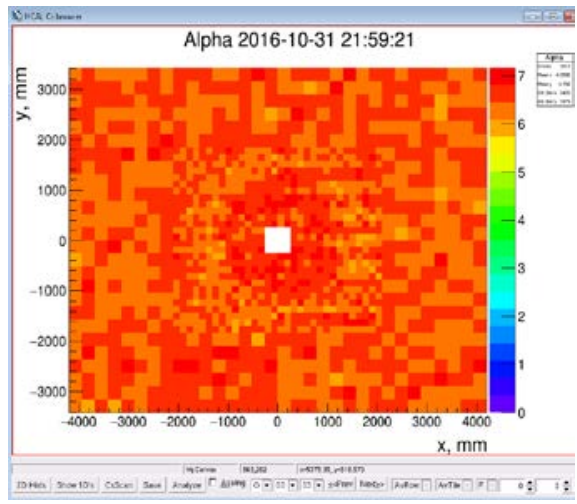

Fig. 18: Multipass calibration results: distributions of $G_{0}(a)$ and $\alpha(b)$ parameters of regulation curve 


\section{$L H C b$

${ }^{137}$ Cs calibration system allows to measure the response $A_{i}$ of every individual scintillating tile row $i$. Therefore degradation of relative light yield $A_{i} / A_{5}$ is measured with respect to a reference ${ }^{137} \mathrm{Cs}$ run at zero luminosity:

$$
R_{i}=\left(\frac{A_{i}}{A_{5}}\right) /\left(\frac{A_{i}^{\text {ref }}}{A_{5}^{\text {ref }}}\right), A_{i}^{\text {ref }} \text { and } \begin{array}{r}
A_{5}^{\text {ref }} \\
- \text { reference amplitudes, from } \\
\text { the }{ }^{137} \text { Cs scan of 29.03.2011 }
\end{array}
$$
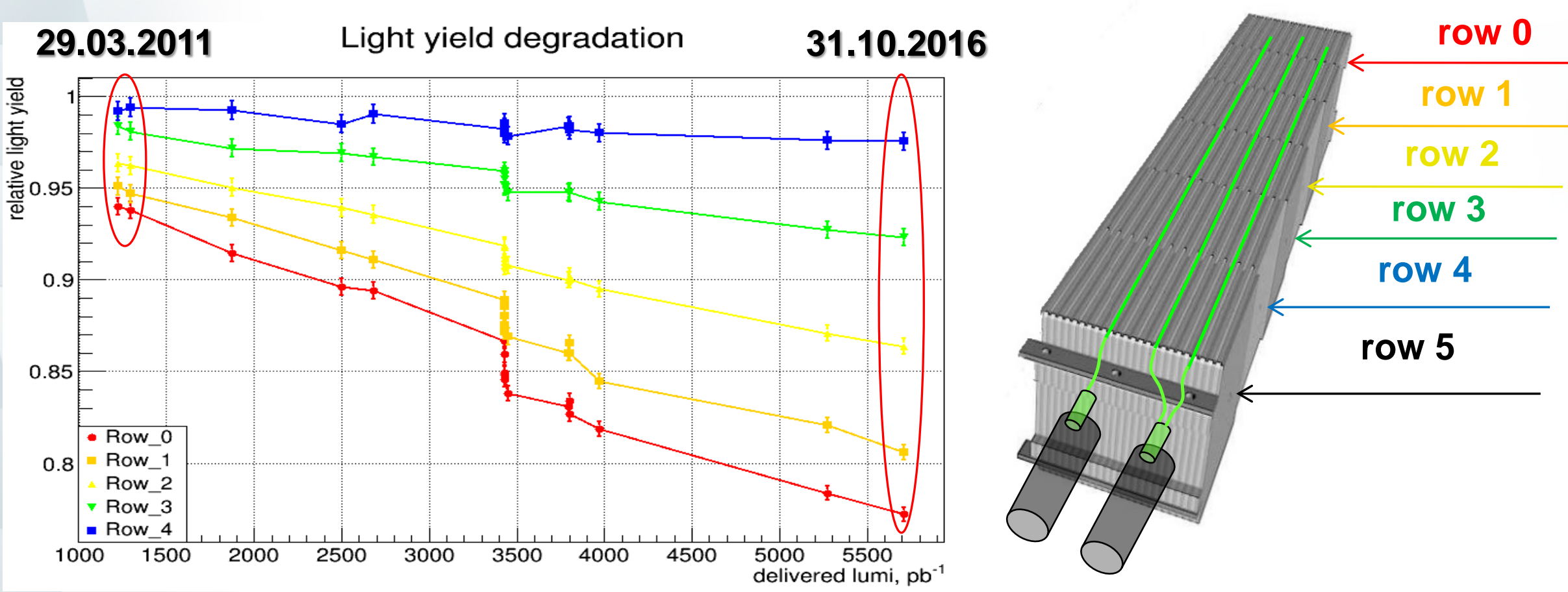

Fig. 19: Light yield degradation; average over 44 central cells 


\section{$\begin{array}{ll}\text { KHCb } & \text { Conclusion }\end{array}$}

\section{ECAL:}

- calibration based on reconstruction of the $\pi^{0}$ meson invariant mass is carried out on a monthly basis;

- allowed to achieve a calibration accuracy of $2-2.5 \%$

\section{HCAL:}

- the cesium calibration system is regularly used for the HCAL calibration starting from the beginning of the LHCb operation in 2008;

- this method provides very detailed information about the calorimeter and allows to measure the response of every individual scintillating tile and the average characteristics of entire cell

The presented methods allow to achieve fast and accurate calibration of ECAL and HCAL.

Calorimeter system is in excellent condition and always ready for work 
Thank you for attention! 